\title{
Field synopsis and systematic meta-analyses of genetic association studies in isolated dystonia
}

Olena Ohlei, MS, a Valerija Dobricic, PhD, a Katja Lohmann, PhD, ${ }^{\text {b }}$ Christine Klein, MD, b Christina Lill, MD, MS, ${ }^{b}$ Lars Bertram, MD, ${ }^{a, c, d d^{*}}$

a Lübeck Interdisciplinary Platform for Genome Analytics, Institutes of Neurogenetics and Cardiogenetics, University of Lübeck, Lübeck, Germany.

b Institute of Neurogenetics, University of Lübeck, Lübeck, Germany.

${ }^{c}$ School of Public Health, Faculty of Medicine, Imperial College, London, UK.

${ }^{\mathrm{d}}$ Dept of Psychology, University of Oslo, Oslo, Norway.

*Correspondence to: Prof. Lars Bertram, Lübeck Interdisciplinary Platform for Genome Analytics, University of Lübeck; Maria-Goeppert-Str. 1, 23562 Lübeck, Germany;

(T): +49 4513101 7491; (F): +49 4513101 7494; Email: lars.bertram@uni-luebeck.de

Key Words: dystonia, field synopsis, meta-analysis, genetic association, genome-wide association study (GWAS)

Funding: This study was supported by the German Research Foundation (DFG grant FOR2488: Main support by subproject "P6" BE2287/6-1 to LB; additional support by subprojects "P7" LI2654/2-1 to CML, "Z1" KL1134/17-1 to CK, and "P4" LO1555/9-1 to KL). 
bioRxiv preprint doi: https://doi.org/10.1101/306522; this version posted April 23,2018 . The copyright holder for this preprint (which was

not certified by peer review) is the author/funder, who has granted bioRxiv a license to display the preprint in perpetuity. It is made available under aCC-BY-NC-ND 4.0 International license.

Ohlei et al, Genetic association studies in dystonia

\section{Abstract}

Background and objectives: Dystonia is a genetically complex disease with both monogenic and polygenic causes. For the latter, numerous genetic associations studies have been performed with largely inconsistent results. The aim of this study was to perform a field synopsis including systematic meta-analyses of genetic association studies in isolated dystonia

Methods: For the field synopsis we systematically screened and scrutinized the published literature using NCBI's PubMed database. For genetic variants with sufficient information in at least two independent datasets, random-effects meta-analyses were performed, including meta-analyses stratified by ethnic descent and dystonia subtypes.

Results: A total of 3,575 articles were identified and scrutinized resulting in the inclusion of 42 independent publications allowing 134 meta-analyses on 45 variants across 17 genes. While our meta-analyses pinpointed several significant association signals with variants in TOR1A, $D R D 1$, and $A R S G$, no single variant displayed compelling association with dystonia in the available data.

Conclusions: Our study provides an up-to-date summary of the status of dystonia genetic association studies. Additional large-scale studies are needed to better understand the genetic causes of isolated dystonia. 
bioRxiv preprint doi: https://doi.org/10.1101/306522; this version posted April 23,2018 . The copyright holder for this preprint (which was not certified by peer review) is the author/funder, who has granted bioRxiv a license to display the preprint in perpetuity. It is made available under aCC-BY-NC-ND 4.0 International license.

Ohlei et al, Genetic association studies in dystonia

\section{Introduction}

Genetically, dystonia is a complex disease with the parallel occurrence of forms with and without causative genetic factors. Over the past decades more than two dozen loci have been proposed as monogenic causes of dystonia[1-3] and 32 confirmed genes (28 with "DYT" labels)[4,5] have been included in the new list of isolated, combined, and complex hereditary dystonia proposed by the International Parkinson and Movement Disorder Society's (MDS) Task Force on Genetic Nomenclature in Movement Disorders[5]. Substantially less is known about the genetic underpinnings of sporadic (i.e. non-monogenic) dystonia. Along these lines, only two GWAS on dystonia subtypes, i.e. musician's dystonia[6] and cervical dystonia[7], have been published to date. The remainder of the existing genetics literature on sporadic dystonia is comprised of candidate gene association studies. These typically focused on genes known to cause familial dystonia as well as functionally founded candidates, such as genes involved in dopamine metabolism or the brain-derived neurotrophic factor (BDNF)[8]. To date, no systematic review covering all genetic polymorphisms investigated in dystonia has been published and only very few meta-analyses utilizing genetic association data exist[9-12]. As a result, it is becoming increasingly difficult to evaluate and interpret the genetics literature pertaining to sporadic dystonia. The aim of this work was to overcome this limitation and perform the first systematic synopsis - including meta-analyses of all available genetic association data - in the dystonia field. To this end, we carefully screened more than 3,500 articles and performed a total of 134 meta-analyses across 52 case-control datasets from 42 independent publications, thereby vastly increasing the number of meta-analyses currently available for dystonia[9-12]. The accrued literature database and meta-analysis results are made available in the supplement of this manuscript. 
Ohlei et al, Genetic association studies in dystonia

\section{Methods}

\section{Literature searches}

\subsection{Systematic literature searches}

Overall, our study followed the approach developed earlier by our group for systematic field synopses in Alzheimer's disease[13] and Parkinson's disease[14]. At the outset, this entailed a systematic literature search using NCBI's "PubMed" database for papers published until January 23, 2018. In a first stage, we searched for keywords "(dystoni* OR (writer* AND cramp) OR graphospasm OR blepharospasm OR torticollis OR meige OR spasmodic OR dysphonia OR retrocollis OR antecollis OR laterocollis OR (musician* AND cramp) OR (occupation* AND cramp) OR (golf* AND cramp) OR yips) AND associat* AND gene*”. In the second stage, we separately searched for each gene identified in stage 1 and for 28 loci with a "DYT" label (Figure1; DYT designations taken from refs. 5 and 15; see Supplementary Tables S1 and S2 for more details). Of note, prior to the revision of the dystonia classification and nomenclature in 2013, isolated dystonia (i.e. pure dystonia with or without dystonic tremor) was referred to as "primary" dystonia, a term mixing phenomenological and etiological features, whose use is no longer recommended[16]. As the clinical part of the definition of "primary" dystonia was identical to that of "isolated" dystonia, we exclusively use the new and unambiguous term "isolated" dystonia for all studies referred to in this article.

\subsection{Inclusion / exclusion criteria}

We only considered publications representing association studies between biallelic genetic polymorphisms (minor allele frequency in included data sets or in the general population $\geq 0.01$ based on ref. 17) and isolated dystonia phenotypes. Additional eligibility criteria were: publication in a peer-reviewed journal, publication in English, and analysis in at least 10 dystonia cases and 10 control subjects. We excluded studies assessing dystonia with a known etiology (previously known as "secondary" dystonia), studies using family-based designs, and 
bioRxiv preprint doi: https://doi.org/10.1101/306522; this version posted April 23, 2018. The copyright holder for this preprint (which was not certified by peer review) is the author/funder, who has granted bioRxiv a license to display the preprint in perpetuity. It is made available under aCC-BY-NC-ND 4.0 International license.

Ohlei et al, Genetic association studies in dystonia

studies on mitochondrial DNA. Furthermore, we excluded studies that contrasted their case groups against "database controls" and those with cases carrying a known dystonia mutation.

\section{Database}

From each eligible article, we extracted a range of informative variables into a Microsoft Excel database (full database in Supplementary Table S2). The initial data entry was performed by O.O. followed by double checking of all variant IDs and genotype distributions by V.D.

\section{Statistical analyses}

For all eligible datasets, we calculated odds ratios (OR) and corresponding 95\% confidence intervals (Cls), assuming an additive genetic model. For datasets with allele frequency information only, we calculated the corresponding genotype distributions assuming HardyWeinberg equilibrium. For four variants, the same or largely overlapping data sets were published in separate articles[18-21]. In these instances, we only included the genotype distributions for the larger of the two datasets, i.e. refs. 20 and 21 . For variants with independent genetic data in at least two non-overlapping datasets we calculated random effects summary ORs using PLINK 1.9[22,23]. Whenever sufficient data were available, metaanalyses were stratified by ethnic descent group in addition to calculating summary ORs across all ethnic groups combined. In addition to calculating meta-analyses across all forms of isolated dystonia, we divided our analyses into focal dystonia (i.e. cervical dystonia, blepharospasm, musician's dystonia, writer's cramp, and spasmodic dysphonia), segmental dystonia, and generalized dystonia. Whenever possible, subgroups were further stratified into groups of differing ethnic descent. Overall, this led to 134 meta-analyses of 45 variants across 17 genes. Pairwise linkage disequilibrium estimates across SNPs within the same $\pm 1 \mathrm{Mb}$ interval in the 1000GP (phase 3) data[17] were calculated using PLINK 1.9 (Supplementary Table S3). This identified 32 independent $\left(r^{2}<0.3\right)$ variants; accordingly, the study-wide significance threshold was set to $\alpha=0.00156(=0.05 / 32)$. Heterogeneity across studies was assessed by calculating the $\mathrm{I}^{2}$ metric $\left(\mathrm{I}^{2}>75 \%\right.$ indicates excess heterogeneity[14]). 
bioRxiv preprint doi: https://doi org/10.1101/306522; this version posted April 23,2018 . The copyright holder for this preprint (which was not certified by peer review) is the author/funder, who has granted bioRxiv a license to display the preprint in perpetuity. It is made available under aCC-BY-NC-ND 4.0 International license.

Ohlei et al, Genetic association studies in dystonia

\section{Results}

\section{Literature search}

The systematic literature search (up-to-date until January 23rd, 2018) identified 3,575 potentially eligible articles (Figure 1). After systematic review of titles, abstracts and full text (as needed) versions of these papers we identified 42 eligible publications (describing association data in 52 independent datasets. Analyzed datasets originated from a total of 17 different countries spread across four continents. In total, 233 polymorphisms across 33 loci were investigated in these 42 publications. Most $(n=40)$ used a candidate gene approach, while two papers were GWAS. The entire database of this field synopsis is provided in Supplementary Table 2.

\section{Meta-analysis results}

For the meta-analyses, we only considered variants with at least two independent nonoverlapping datasets with available genotype or allele data. This included screening 557,621 SNPs from a musician's dystonia GWAS dataset[6] for an overlap with the 233 polymorphisms identified in the literature screen. Overall, this procedure resulted in sufficient data for a total of 45 variants across 17 genetic loci (median sample size per data set: 458 , range $88-5,385$ ). Of these, 42 variants could be analysed after stratification in for datasets of Caucasian descent, and 6 after stratification for Asian descent. Only five variants in TOR1A, DRD1 and ARSG showed nominally significant evidence for association: i.e. rs4532 (OR [95 \% Cl]: 1.37 [1.131.67], $P=0.00153$ ), rs35153737 (OR [95\% Cl]: 1.46 [1.14-1.88], $P=0.00315)$, rs 13283584 (OR [95\% Cl]: 1.32 [1.08-1.61], $\mathrm{P}=0.00584$ ), rs7342975 (OR [95 \% Cl]: 2.00 [1.15-3.47], $\mathrm{P}=0.00572$ ), rs9972951 (OR [95 \% Cl]: 2.16 [1.06-4.40], $\mathrm{P}=0.0342$ ), but only variant rs4532 in $D R D 1$ survived correction for multiple testing. Meta-analyses in subgroups stratified by ethnic descent did not reveal any additional associations (Table 1).

Meta-analyses divided by diagnostic subgroup are summarized in Table 2 and yielded five nominally significant results, two of which were not observed in the analyses without diagnostic 
bioRxiv preprint doi: https://doi.org/10.1101/306522; this version posted April 23, 2018. The copyright holder for this preprint (which was not certified by peer review) is the author/funder, who has granted bioRxiv a license to display the preprint in perpetuity. It is made available under aCC-BY-NC-ND 4.0 International license.

Ohlei et al, Genetic association studies in dystonia

stratification, i.e. rs1801968 in TOR1A (OR [95\% C.I.]: 3.10 [1.25-7.68], $\mathrm{P}=0.0142$ ), associated with writer's cramp, and rs11655081 in ARSG (OR [95\% C.I.]: 4.42 [2.72-7.19], $\mathrm{P}=2.11 \mathrm{e}-09$, respectively) associated with musician's dystonia (Table 2). While the latter finding surpassed the threshold of study- and genome-wide significance, we note that this meta-analysis is only based on two independent datasets, both of which originate from the same (and only) paper on the topic[6]. The same variant was also assessed in independent datasets with other focal (i.e. cervical, blepharospasm; Table 2B, D) and segmental (Table 2G) forms of dystonia but did not show any evidence for association with these dystonia subtypes[6].

\section{Discussion}

This work represents the first systematic field synopsis of genetic association studies in dystonia. Overall, we present the results of 134 meta-analyses on 45 variants across 17 genes. While nominal associations with several loci were observed, only variants in $D R D 1$ and $A R S G$ survived multiple testing correction. However, even these two most significant results should be interpreted with caution owing to comparatively small sample size.

Prior to this study meta-analyses on genetic association data in dystonia were only available for two genes, i.e. TOR1A[9,10] and $B D N F 11,12]$, both with conflicting results, i.e. one study interpreted their results in favor of a role in dystonia susceptibility, while the other study reached an opposite conclusion. Although our results provide some degree of support for an involvement of TOR1A SNP rs35153737 in Asians and rs13283584 in Caucasians (Table 1), no association evidence was observed with the only available (but widely tested) SNP (rs6265) in BDNF.

One notable observation and important limitation of our study is that most meta-analyses are based on relatively small sample sizes (median combined sample size of across all metaanalyses was only 2,882 individuals, compared to $\sim 4,500$ for Alzheimer's[13] and $\sim 7,500$ for Parkinson's disease[14]). Provided that the genetic liability of dystonia is mostly governed by 
bioRxiv preprint doi: https://doi.org/10.1101/306522; this version posted April 23,2018 . The copyright holder for this preprint (which was

not certified by peer review) is the author/funder, who has granted bioRxiv a license to display the preprint in perpetuity. It is made available under aCC-BY-NC-ND 4.0 International license.

Ohlei et al, Genetic association studies in dystonia

small effect variants with ORs $<1.2$, our power to detect such associations was small, and only efforts with substantially increased sample sizes for both patients and controls will have sufficient power to detect such effect sizes[14]. Other limitations of our study relate to the search strategy, which might not have identified some eligible papers, and an erroneous data extraction and representation procedure. However, while such errors are unavoidable, our ample experience in the collection, annotation and curation of genetic association data in the context of systematic field synopses[13,14,24-27] suggest that these errors will likely be infrequent and will not affect the major conclusion(s) of our paper.

Notwithstanding these and possibly other unrecognized limitations, our study represents the first systematic synopsis of genetic association studies in dystonia and the largest collection of meta-analyses in the field. Despite its comprehensiveness, and in contrast to genetic studies on monogenic forms of the disease, it currently provides only little support for an existence of strong genetic risk factors acting in sporadic dystonia. This situation will likely change upon completion of additional large-scale GWAS of sufficient size, which will hopefully provide new insights into the pathogenetic forces underlying the onset and progression of this debilitating disease. 
bioRxiv preprint doi: https://doi.org/10.1101/306522; this version posted April 23, 2018. The copyright holder for this preprint (which was not certified by peer review) is the author/funder, who has granted bioRxiv a license to display the preprint in perpetuity. It is made available under aCC-BY-NC-ND 4.0 International license.

Ohlei et al, Genetic association studies in dystonia

\section{Acknowledgements}

This study was supported by by the German Research Foundation (DFG) (FOR2488, main support by subproject "P6" BE2287/6-1 to LB, additional support by subprojects "P7" LI2654/21 to CML, "Z1" KL1134/17-1 to CK, and "P4" LO1555/9-1 to KL). CK is the recipient of a career development award from the Hermann and Lilly Schilling Foundation. The authors report no conflict of interest. 
Ohlei et al, Genetic association studies in dystonia

\section{References}

[1] H.A. Jinnah, J.K. Teller, W.R. Galpern, Recent Developments in Dystonia, (n.d.). doi:10.1097/WCO.0000000000000213.

[2] C. Klein, Genetics in dystonia, Parkinsonism Relat. Disord. 20 (2014) S137-S142. doi:10.1016/S1353-8020(13)70033-6.

[3] K. Lohmann, C. Klein, Update on the Genetics of Dystonia, (1910). doi:10.1007/s11910-017-0735-0.

[4] C. Klein, K. Lohmann, C. Marras, A. Münchau, Hereditary Dystonia Overview, University of Washington, Seattle, 1993. http://www.ncbi.nlm.nih.gov/pubmed/20301334 (accessed February 15, 2018).

[5] C. Marras, A. Lang, B.P. van de Warrenburg, C.M. Sue, S.J. Tabrizi, L. Bertram, S. Mercimek-Mahmutoglu, D. Ebrahimi-Fakhari, T.T. Warner, A. Durr, B. Assmann, K. Lohmann, V. Kostic, C. Klein, Nomenclature of genetic movement disorders: Recommendations of the international Parkinson and movement disorder society task force, Mov. Disord. 31 (2016) 436-457. doi:10.1002/mds.26527.

[6] K. Lohmann, A. Schmidt, A. Schillert, S. Winkler, A. Albanese, F. Baas, A.R. Bentivoglio, F. Borngräber, N. Brüggemann, G. Defazio, F. Del Sorbo, G. Deuschl, M.J. Edwards, T. Gasser, P. Gómez-Garre, J. Graf, J.L. Groen, A. Grünewald, J. Hagenah, C. Hemmelmann, H.C. Jabusch, R. Kaji, M. Kasten, H. Kawakami, V.S. Kostic, M. Liguori, P. Mir, A. Münchau, F. Ricchiuti, S. Schreiber, K. Siegesmund, M. Svetel, M.A.J. Tijssen, E.M. Valente, A. Westenberger, K.E. Zeuner, S. Zittel, E. Altenmüller, A. Ziegler, C. Klein, Genome-wide association study in musician's dystonia: A risk variant at the arylsulfatase G locus?, Mov. Disord. 29 (2014) 921-927. doi:10.1002/mds.25791.

[7] K.Y. Mok, S.A. Schneider, D. Trabzuni, M. Stamelou, M. Edwards, D. Kasperaviciute, S. Pickering-Brown, M. Silverdale, J. Hardy, K.P. Bhatia, Genomewide association 
Ohlei et al, Genetic association studies in dystonia

study in cervical dystonia demonstrates possible association with sodium leak channel., Mov. Disord. 29 (2014) 245-51. doi:10.1002/mds.25732.

[8] K. Lohmann, C. Klein, Genetics of dystonia: What's known? What's new? What's next?, Mov. Disord. 28 (2013) 899-905. doi:10.1002/mds.25536.

[9] J.L. Groen, K. Ritz, M.W. Tanck, B.P. van de Warrenburg, J.J. van Hilten, M. Aramideh, F. Baas, M.A.J. Tijssen, Is TOR1A a risk factor in adult-onset primary torsion dystonia?, Mov. Disord. 28 (2013) 827-31. doi:10.1002/mds.25381.

[10] V. Siokas, E. Dardiotis, E.E. Tsironi, G. Tsivgoulis, D. Rikos, M. Sokratous, S. Koutsias, K. Paterakis, G. Deretzi, G.M. Hadjigeorgiou, The Role of TOR1A Polymorphisms in Dystonia: A Systematic Review and Meta-Analysis., PLoS One. 12 (2017) e0169934. doi:10.1371/journal.pone.0169934.

[11] P. Gómez-Garre, I. Huertas-Fernández, M.T. Cáceres-Redondo, A. Alonso-Canovas, I. Bernal-Bernal, A. Blanco-Ollero, M. Bonilla-Toribio, J.A. Burguera, M. Carballo, F. Carrillo, M.J. Catalán-Alonso, F. Escamilla-Sevilla, R. Espinosa-Rosso, M.C. Fernández-Moreno, J. García-Caldentey, J.M. García-Moreno, P.J. García-Ruiz, S. Giacometti-Silveira, J. Gutiérrez-García, S. Jesús, E. López-Valdés, J.C. MartínezCastrillo, I. Martínez-Torres, M.P. Medialdea-Natera, C. Méndez-Lucena, A. MínguezCastellanos, M. Moya, J.J. Ochoa-Sepulveda, T. Ojea, N. Rodríguez, M. SilleroSánchez, L. Vargas-González, P. Mir, BDNF Val66Met polymorphism in primary adultonset dystonia: A case-control study and meta-analysis, Mov. Disord. 29 (2014) 10831086. doi:10.1002/mds.25938.

[12] W. Sako, N. Murakami, Y. Izumi, R. Kaji, Val66Met polymorphism of brain-derived neurotrophic factor is associated with idiopathic dystonia., J. Clin. Neurosci. 22 (2015) 575-7. doi:10.1016/j.jocn.2014.08.014.

[13] L. Bertram, M.B. McQueen, K. Mullin, D. Blacker, R.E. Tanzi, Systematic metaanalyses of Alzheimer disease genetic association studies: the AlzGene database, 
Ohlei et al, Genetic association studies in dystonia

Nat. Genet. 39 (2007) 17-23. doi:10.1038/ng1934.

[14] C.M. Lill, J.T. Roehr, M.B. McQueen, F.K. Kavvoura, S. Bagade, B.-M.M. Schjeide,

L.M. Schjeide, E. Meissner, U. Zauft, N.C. Allen, T. Liu, M. Schilling, K.J. Anderson, G.

Beecham, D. Berg, J.M. Biernacka, A. Brice, A.L. DeStefano, C.B. Do, N. Eriksson,

S.A. Factor, M.J. Farrer, T. Foroud, T. Gasser, T. Hamza, J.A. Hardy, P. Heutink, E.M.

Hill-Burns, C. Klein, J.C. Latourelle, D.M. Maraganore, E.R. Martin, M. Martinez, R.H.

Myers, M.A. Nalls, N. Pankratz, H. Payami, W. Satake, W.K. Scott, M. Sharma, A.B.

Singleton, K. Stefansson, T. Toda, J.Y. Tung, J. Vance, N.W. Wood, C.P. Zabetian, P.

23andMe Genetic Epidemiology of Parkinson's Disease Consortium, R.E. International

Parkinson's Disease Genomics Consortium, M.J. Parkinson's Disease GWAS

Consortium, F. Wellcome Trust Case Control Consortium 2), P. Young, R.E. Tanzi,

M.J. Khoury, F. Zipp, H. Lehrach, J.P.A. Ioannidis, L. Bertram, Comprehensive

research synopsis and systematic meta-analyses in Parkinson's disease genetics: The PDGene database., PLoS Genet. 8 (2012) e1002548.

doi:10.1371/journal.pgen.1002548.

[15] E. Meyer, K.J. Carss, J. Rankin, J.M.E. Nichols, D. Grozeva, A.P. Joseph, N.E.

Mencacci, A. Papandreou, J. Ng, S. Barral, A. Ngoh, H. Ben-Pazi, M.A. Willemsen, D.

Arkadir, A. Barnicoat, H. Bergman, S. Bhate, A. Boys, N. Darin, N. Foulds, N.

Gutowski, A. Hills, H. Houlden, J.A. Hurst, Z. Israel, M. Kaminska, P. Limousin, D.

Lumsden, S. McKee, S. Misra, S.S. Mohammed, V. Nakou, J. Nicolai, M. Nilsson, H.

Pall, K.J. Peall, G.B. Peters, P. Prabhakar, M.S. Reuter, P. Rump, R. Segel, M.

Sinnema, M. Smith, P. Turnpenny, S.M. White, D. Wieczorek, S. Wiethoff, B.T.

Wilson, G. Winter, C. Wragg, S. Pope, S.J.H. Heales, D. Morrogh, A. Pittman, L.J.

Carr, B. Perez-Dueñas, J.-P. Lin, A. Reis, W.A. Gahl, C. Toro, K.P. Bhatia, N.W.

Wood, E.-J. Kamsteeg, W.K. Chong, P. Gissen, M. Topf, R.C. Dale, J.R. Chubb, F.L.

Raymond, M.A. Kurian, Mutations in the histone methyltransferase gene KMT2B

cause complex early-onset dystonia, Nat. Genet. 49 (2017) 223-237. 
bioRxiv preprint doi: https://doi.org/10.1101/306522; this version posted April 23, 2018. The copyright holder for this preprint (which was not certified by peer review) is the author/funder, who has granted bioRxiv a license to display the preprint in perpetuity. It is made available under aCC-BY-NC-ND 4.0 International license.

Ohlei et al, Genetic association studies in dystonia

doi:10.1038/ng.3740.

[16] A. Albanese, K. Bhatia, S.B. Bressman, M.R. Delong, S. Fahn, V.S.C. Fung, M. Hallett, J. Jankovic, H.A. Jinnah, C. Klein, A.E. Lang, J.W. Mink, J.K. Teller, Phenomenology and classification of dystonia: a consensus update., Mov. Disord. 28 (2013) 863-73. doi:10.1002/mds.25475.

[17] A. Auton, G.R. Abecasis, D.M. Altshuler, R.M. Durbin, G.R. Abecasis, D.R. Bentley, A. Chakravarti, A.G. Clark, P. Donnelly, E.E. Eichler, P. Flicek, S.B. Gabriel, R.A. Gibbs, E.D. Green, M.E. Hurles, B.M. Knoppers, J.O. Korbel, E.S. Lander, C. Lee, H. Lehrach, E.R. Mardis, G.T. Marth, G.A. McVean, D.A. Nickerson, J.P. Schmidt, S.T. Sherry, J. Wang, R.K. Wilson, R.A. Gibbs, E. Boerwinkle, H. Doddapaneni, Y. Han, V. Korchina, C. Kovar, S. Lee, D. Muzny, J.G. Reid, Y. Zhu, J. Wang, Y. Chang, Q. Feng, X. Fang, X. Guo, M. Jian, H. Jiang, X. Jin, T. Lan, G. Li, J. Li, Y. Li, S. Liu, X. Liu, Y. Lu, X. Ma, M. Tang, B. Wang, G. Wang, H. Wu, R. Wu, X. Xu, Y. Yin, D. Zhang, W. Zhang, J. Zhao, M. Zhao, X. Zheng, E.S. Lander, D.M. Altshuler, S.B. Gabriel, N. Gupta, N. Gharani, L.H. Toji, N.P. Gerry, A.M. Resch, P. Flicek, J. Barker, L. Clarke, L. Gil, S.E. Hunt, G. Kelman, E. Kulesha, R. Leinonen, W.M. McLaren, R.

Radhakrishnan, A. Roa, D. Smirnov, R.E. Smith, I. Streeter, A. Thormann, I. Toneva, B. Vaughan, X. Zheng-Bradley, D.R. Bentley, R. Grocock, S. Humphray, T. James, Z. Kingsbury, H. Lehrach, R. Sudbrak, M.W. Albrecht, V.S. Amstislavskiy, T.A. Borodina, M. Lienhard, F. Mertes, M. Sultan, B. Timmermann, M.-L. Yaspo, E.R. Mardis, R.K. Wilson, L. Fulton, R. Fulton, S.T. Sherry, V. Ananiev, Z. Belaia, D. Beloslyudtsev, N. Bouk, C. Chen, D. Church, R. Cohen, C. Cook, J. Garner, T. Hefferon, M. Kimelman, C. Liu, J. Lopez, P. Meric, C. O’Sullivan, Y. Ostapchuk, L. Phan, S. Ponomarov, V. Schneider, E. Shekhtman, K. Sirotkin, D. Slotta, H. Zhang, G.A. McVean, R.M. Durbin, S. Balasubramaniam, J. Burton, P. Danecek, T.M. Keane, A. Kolb-Kokocinski, S. McCarthy, J. Stalker, M. Quail, J.P. Schmidt, C.J. Davies, J. Gollub, T. Webster, B. Wong, Y. Zhan, A. Auton, C.L. Campbell, Y. Kong, A. Marcketta, R.A. Gibbs, F. Yu, L. 
Antunes, M. Bainbridge, D. Muzny, A. Sabo, Z. Huang, J. Wang, L.J.M. Coin, L. Fang, X. Guo, X. Jin, G. Li, Q. Li, Y. Li, Z. Li, H. Lin, B. Liu, R. Luo, H. Shao, Y. Xie, C. Ye, C. Yu, F. Zhang, H. Zheng, H. Zhu, C. Alkan, E. Dal, F. Kahveci, G.T. Marth, E.P. Garrison, D. Kural, W.-P. Lee, W. Fung Leong, M. Stromberg, A.N. Ward, J. Wu, M. Zhang, M.J. Daly, M.A. DePristo, R.E. Handsaker, D.M. Altshuler, E. Banks, G. Bhatia, G. del Angel, S.B. Gabriel, G. Genovese, N. Gupta, H. Li, S. Kashin, E.S. Lander, S.A. McCarroll, J.C. Nemesh, R.E. Poplin, S.C. Yoon, J. Lihm, V. Makarov, A.G. Clark, S. Gottipati, A. Keinan, J.L. Rodriguez-Flores, J.O. Korbel, T. Rausch, M.H. Fritz, A.M. Stütz, P. Flicek, K. Beal, L. Clarke, A. Datta, J. Herrero, W.M. McLaren, G.R.S. Ritchie, R.E. Smith, D. Zerbino, X. Zheng-Bradley, P.C. Sabeti, I. Shlyakhter, S.F. Schaffner, J. Vitti, D.N. Cooper, E. V. Ball, P.D. Stenson, D.R. Bentley, B. Barnes, M. Bauer, R. Keira Cheetham, A. Cox, M. Eberle, S. Humphray, S. Kahn, L. Murray, J. Peden, R. Shaw, E.E. Kenny, M.A. Batzer, M.K. Konkel, J.A. Walker, D.G. MacArthur, M. Lek, R. Sudbrak, V.S. Amstislavskiy, R. Herwig, E.R. Mardis, L. Ding, D.C. Koboldt, D. Larson, K. Ye, S. Gravel, A. Swaroop, E. Chew, T. Lappalainen, Y. Erlich, M. Gymrek, T. Frederick Willems, J.T. Simpson, M.D. Shriver, J.A. Rosenfeld, C.D. Bustamante, S.B. Montgomery, F.M. De La Vega, J.K. Byrnes, A.W. Carroll, M.K. DeGorter, P. Lacroute, B.K. Maples, A.R. Martin, A. Moreno-Estrada, S.S. Shringarpure, F. Zakharia, E. Halperin, Y. Baran, C. Lee, E. Cerveira, J. Hwang, A. Malhotra, D. Plewczynski, K. Radew, M. Romanovitch, C. Zhang, F.C.L. Hyland, D.W. Craig, A. Christoforides, N. Homer, T. Izatt, A.A. Kurdoglu, S.A. Sinari, K. Squire, S.T. Sherry, C. Xiao, J. Sebat, D. Antaki, M. Gujral, A. Noor, K. Ye, E.G. Burchard, R.D. Hernandez, C.R. Gignoux, D. Haussler, S.J. Katzman, W. James Kent, B. Howie, A. Ruiz-Linares, E.T. Dermitzakis, S.E. Devine, G.R. Abecasis, H. Min Kang, J.M. Kidd, T. Blackwell, S. Caron, W. Chen, S. Emery, L. Fritsche, C. Fuchsberger, G. Jun, B. Li, R. Lyons, C. Scheller, C. Sidore, S. Song, E. Sliwerska, D. Taliun, A. Tan, R. Welch, M. Kate Wing, X. Zhan, P. Awadalla, A. Hodgkinson, Y. Li, X. Shi, A. Quitadamo, G. Lunter, G.A. McVean, J.L. Marchini, S. Myers, C. Churchhouse, O. Delaneau, A. 
Gupta-Hinch, W. Kretzschmar, Z. Iqbal, I. Mathieson, A. Menelaou, A. Rimmer, D.K. Xifara, T.K. Oleksyk, Y. Fu, X. Liu, M. Xiong, L. Jorde, D. Witherspoon, J. Xing, E.E. Eichler, B.L. Browning, S.R. Browning, F. Hormozdiari, P.H. Sudmant, E. Khurana, R.M. Durbin, M.E. Hurles, C. Tyler-Smith, C.A. Albers, Q. Ayub, S. Balasubramaniam, Y. Chen, V. Colonna, P. Danecek, L. Jostins, T.M. Keane, S. McCarthy, K. Walter, Y. Xue, M.B. Gerstein, A. Abyzov, S. Balasubramanian, J. Chen, D. Clarke, Y. Fu, A.O. Harmanci, M. Jin, D. Lee, J. Liu, X. Jasmine Mu, J. Zhang, Y. Zhang, Y. Li, R. Luo, H. Zhu, C. Alkan, E. Dal, F. Kahveci, G.T. Marth, E.P. Garrison, D. Kural, W.-P. Lee, A.N. Ward, J. Wu, M. Zhang, S.A. McCarroll, R.E. Handsaker, D.M. Altshuler, E. Banks, G. del Angel, G. Genovese, C. Hartl, H. Li, S. Kashin, J.C. Nemesh, K. Shakir, S.C. Yoon, J. Lihm, V. Makarov, J. Degenhardt, J.O. Korbel, M.H. Fritz, S. Meiers, B. Raeder, T. Rausch, A.M. Stütz, P. Flicek, F. Paolo Casale, L. Clarke, R.E. Smith, O. Stegle, X. Zheng-Bradley, D.R. Bentley, B. Barnes, R. Keira Cheetham, M. Eberle, S. Humphray, S. Kahn, L. Murray, R. Shaw, E.-W. Lameijer, M.A. Batzer, M.K. Konkel, J.A. Walker, L. Ding, I. Hall, K. Ye, P. Lacroute, C. Lee, E. Cerveira, A. Malhotra, J. Hwang, D. Plewczynski, K. Radew, M. Romanovitch, C. Zhang, D.W. Craig, N. Homer, D. Church, C. Xiao, J. Sebat, D. Antaki, V. Bafna, J. Michaelson, K. Ye, S.E. Devine, E.J. Gardner, G.R. Abecasis, J.M. Kidd, R.E. Mills, G. Dayama, S. Emery, G. Jun, X. Shi, A. Quitadamo, G. Lunter, G.A. McVean, K. Chen, X. Fan, Z. Chong, T. Chen, D. Witherspoon, J. Xing, E.E. Eichler, M.J. Chaisson, F. Hormozdiari, J. Huddleston, M. Malig, B.J. Nelson, P.H. Sudmant, N.F. Parrish, E. Khurana, M.E. Hurles, B.

Blackburne, S.J. Lindsay, Z. Ning, K. Walter, Y. Zhang, M.B. Gerstein, A. Abyzov, J. Chen, D. Clarke, H. Lam, X. Jasmine Mu, C. Sisu, J. Zhang, Y. Zhang, R.A. Gibbs, F. Yu, M. Bainbridge, D. Challis, U.S. Evani, C. Kovar, J. Lu, D. Muzny, U. Nagaswamy, J.G. Reid, A. Sabo, J. Yu, X. Guo, W. Li, Y. Li, R. Wu, G.T. Marth, E.P. Garrison, W. Fung Leong, A.N. Ward, G. del Angel, M.A. DePristo, S.B. Gabriel, N. Gupta, C. Hartl, R.E. Poplin, A.G. Clark, J.L. Rodriguez-Flores, P. Flicek, L. Clarke, R.E. Smith, X. Zheng-Bradley, D.G. MacArthur, E.R. Mardis, R. Fulton, D.C. Koboldt, S. Gravel, C.D. 
Ohlei et al, Genetic association studies in dystonia

Bustamante, D.W. Craig, A. Christoforides, N. Homer, T. Izatt, S.T. Sherry, C. Xiao, E.T. Dermitzakis, G.R. Abecasis, H. Min Kang, G.A. McVean, M.B. Gerstein, S. Balasubramanian, L. Habegger, H. Yu, P. Flicek, L. Clarke, F. Cunningham, I. Dunham, D. Zerbino, X. Zheng-Bradley, K. Lage, J. Berg Jespersen, H. Horn, S.B. Montgomery, M.K. DeGorter, E. Khurana, C. Tyler-Smith, Y. Chen, V. Colonna, Y. Xue, M.B. Gerstein, S. Balasubramanian, Y. Fu, D. Kim, A. Auton, A. Marcketta, R. Desalle, A. Narechania, M.A. Wilson Sayres, E.P. Garrison, R.E. Handsaker, S. Kashin, S.A. McCarroll, J.L. Rodriguez-Flores, P. Flicek, L. Clarke, X. Zheng-Bradley, Y. Erlich, M. Gymrek, T. Frederick Willems, C.D. Bustamante, F.L. Mendez, G. David Poznik, P.A. Underhill, C. Lee, E. Cerveira, A. Malhotra, M. Romanovitch, C. Zhang, G.R. Abecasis, L. Coin, H. Shao, D. Mittelman, C. Tyler-Smith, Q. Ayub, R. Banerjee, M. Cerezo, Y. Chen, T.W. Fitzgerald, S. Louzada, A. Massaia, S. McCarthy, G.R. Ritchie, Y. Xue, F. Yang, R.A. Gibbs, C. Kovar, D. Kalra, W. Hale, D. Muzny, J.G. Reid, J. Wang, X. Dan, X. Guo, G. Li, Y. Li, C. Ye, X. Zheng, D.M. Altshuler, P. Flicek, L. Clarke, X. Zheng-Bradley, D.R. Bentley, A. Cox, S. Humphray, S. Kahn, R. Sudbrak, M.W. Albrecht, M. Lienhard, D. Larson, D.W. Craig, T. Izatt, A.A. Kurdoglu, S.T. Sherry, C. Xiao, D. Haussler, G.R. Abecasis, G.A. McVean, R.M. Durbin, S. Balasubramaniam, T.M. Keane, S. McCarthy, J. Stalker, A. Chakravarti, B.M. Knoppers, G.R. Abecasis, K.C. Barnes, C. Beiswanger, E.G. Burchard, C.D. Bustamante, H. Cai, H. Cao, R.M. Durbin, N.P. Gerry, N. Gharani, R.A. Gibbs, C.R. Gignoux, S. Gravel, B. Henn, D. Jones, L. Jorde, J.S. Kaye, A. Keinan, A. Kent, A. Kerasidou, Y. Li, R. Mathias, G.A. McVean, A. Moreno-Estrada, P.N. Ossorio, M. Parker, A.M. Resch, C.N. Rotimi, C.D. Royal, K. Sandoval, Y. Su, R. Sudbrak, Z. Tian, S. Tishkoff, L.H. Toji, C. Tyler-Smith, M. Via, Y. Wang, H. Yang, L. Yang, J. Zhu, W. Bodmer, G. Bedoya, A. Ruiz-Linares, Z. Cai, Y. Gao, J. Chu, L. Peltonen, A. GarciaMontero, A. Orfao, J. Dutil, J.C. Martinez-Cruzado, T.K. Oleksyk, K.C. Barnes, R.A. Mathias, A. Hennis, H. Watson, C. McKenzie, F. Qadri, R. LaRocque, P.C. Sabeti, J. Zhu, X. Deng, P.C. Sabeti, D. Asogun, O. Folarin, C. Happi, O. Omoniwa, M. 
Ohlei et al, Genetic association studies in dystonia

Stremlau, R. Tariyal, M. Jallow, F. Sisay Joof, T. Corrah, K. Rockett, D. Kwiatkowski, J. Kooner, T. Tịnh Hiền, S.J. Dunstan, N. Thuy Hang, R. Fonnie, R. Garry, L. Kanneh, L. Moses, P.C. Sabeti, J. Schieffelin, D.S. Grant, C. Gallo, G. Poletti, D. Saleheen, A. Rasheed, L.D. Brooks, A.L. Felsenfeld, J.E. McEwen, Y. Vaydylevich, E.D. Green, A. Duncanson, M. Dunn, J.A. Schloss, J. Wang, H. Yang, A. Auton, L.D. Brooks, R.M. Durbin, E.P. Garrison, H. Min Kang, J.O. Korbel, J.L. Marchini, S. McCarthy, G.A. McVean, G.R. Abecasis, A global reference for human genetic variation, Nature. 526 (2015) 68-74. doi:10.1038/nature15393.

[18] J.R.B. Newman, G.T. Sutherland, R.S. Boyle, N. Limberg, S. Blum, J.D. O'Sullivan, P.A. Silburn, G.D. Mellick, Common polymorphisms in dystonia-linked genes and susceptibility to the sporadic primary dystonias., Parkinsonism Relat. Disord. 18 (2012) 351-7. doi:10.1016/j.parkreldis.2011.11.024.

[19] A. Djarmati, S.A. Schneider, K. Lohmann, S. Winkler, H. Pawlack, J. Hagenah, N. Brüggemann, S. Zittel, T. Fuchs, A. Raković, A. Schmidt, H.-C. Jabusch, R. Wilcox, V.S. Kostić, H. Siebner, E. Altenmüller, A. Münchau, L.J. Ozelius, C. Klein, Mutations in THAP1 (DYT6) and generalised dystonia with prominent spasmodic dysphonia: a genetic screening study, Lancet Neurol. 8 (2009) 447-452. doi:10.1016/S14744422(09)70083-3.

[20] J.R.B. Newman, M. Todorovic, P.A. Silburn, G.T. Sutherland, G.D. Mellick, Lack of reproducibility in re-evaluating associations between $\mathrm{GCH} 1$ polymorphisms and Parkinson's disease and isolated dystonia in an Australian case-control group, Parkinsonism Relat. Disord. 20 (2014) 668-670. doi:10.1016/j.parkreldis.2014.02.014.

[21] K. Lohmann, N. Uflacker, A. Erogullari, T. Lohnau, S. Winkler, A. Dendorfer, S.A. Schneider, A. Osmanovic, M. Svetel, A. Ferbert, S. Zittel, A.A. Kühn, A. Schmidt, E. Altenmüller, A. Münchau, C. Kamm, M. Wittstock, A. Kupsch, E. Moro, J. Volkmann, V. Kostic, F.J. Kaiser, C. Klein, N. Brüggemann, Identification and functional analysis of novel THAP1 mutations., Eur. J. Hum. Genet. 20 (2012) 171-5. 
Ohlei et al, Genetic association studies in dystonia

doi:10.1038/ejhg.2011.159.

[22] S. Purcell, B. Neale, K. Todd-Brown, L. Thomas, M.A.R. Ferreira, D. Bender, J. Maller, P. Sklar, P.I.W. de Bakker, M.J. Daly, P.C. Sham, PLINK: a tool set for whole-genome association and population-based linkage analyses., Am. J. Hum. Genet. 81 (2007) 559-75. doi:10.1086/519795.

[23] C.C. Chang, C.C. Chow, L.C. Tellier, S. Vattikuti, S.M. Purcell, J.J. Lee, Secondgeneration PLINK: rising to the challenge of larger and richer datasets, Gigascience. 4 (2015) 7. doi:10.1186/s13742-015-0047-8.

[24] N.C. Allen, S. Bagade, M.B. McQueen, J.P.A. loannidis, F.K. Kavvoura, M.J. Khoury, R.E. Tanzi, L. Bertram, Systematic meta-analyses and field synopsis of genetic association studies in schizophrenia: the SzGene database., Nat. Genet. 40 (2008) 827-34. doi:10.1038/ng.171.

[25] P.J. Castaldi, M.H. Cho, M. Cohn, F. Langerman, S. Moran, N. Tarragona, H. Moukhachen, R. Venugopal, D. Hasimja, E. Kao, B. Wallace, C.P. Hersh, S. Bagade, L. Bertram, E.K. Silverman, T.A. Trikalinos, The COPD genetic association compendium: a comprehensive online database of COPD genetic associations., Hum. Mol. Genet. 19 (2010) 526-34. doi:10.1093/hmg/ddp519.

[26] F. Chatzinasiou, C.M. Lill, K. Kypreou, I. Stefanaki, V. Nicolaou, G. Spyrou, E. Evangelou, J.T. Roehr, E. Kodela, A. Katsambas, H. Tsao, J.P.A. loannidis, L. Bertram, A.J. Stratigos, Comprehensive Field Synopsis and Systematic Meta-analyses of Genetic Association Studies in Cutaneous Melanoma, JNCI J. Natl. Cancer Inst. 103 (2011) 1227-1235. doi:10.1093/jnci/djr219.

[27] C.M. Lill, O. Abel, L. Bertram, A. Al-Chalabi, Keeping up with genetic discoveries in amyotrophic lateral sclerosis: the ALSoD and ALSGene databases., Amyotroph. Lateral Scler. 12 (2011) 238-49. doi:10.3109/17482968.2011.584629.

[28] B. Yates, B. Braschi, K.A. Gray, R.L. Seal, S. Tweedie, E.A. Bruford, Genenames.org: 
bioRxiv preprint doi: https://doi.org/10.1101/306522; this version posted April 23, 2018. The copyright holder for this preprint (which was not certified by peer review) is the author/funder, who has granted bioRxiv a license to display the preprint in perpetuity. It is made available under aCC-BY-NC-ND 4.0 International license.

Ohlei et al, Genetic association studies in dystonia

the HGNC and VGNC resources in 2017., Nucleic Acids Res. 45 (2017) D619-D625.

doi:10.1093/nar/gkw1033. 
Ohlei et al, Genetic association studies in dystonia

\section{Legends to Tables and Figures}

\section{Main Text:}

Table 1. Results of random-effects meta-analyses based on an additive model in all types of isolated dystonia. $\ddagger \mathrm{C}=$ Of Caucasian descent (i.e. more than $90 \%$ of individuals in a data set belongs to this descent group); $\mathrm{A}=$ Asian descent; All = All ethnic groups combined (see Table S2 for study-specific details).

Table 2. Results of random-effects meta-analyses based on an additive model in diagnostic subgroups of isolated dystonia: a) all types of focal dystonia, b) musician's dystonia, c) blepharospasm, d) cervical dystonia, e) spasmodic dystonia, f) writer's dystonia, g) segmental dystonia, h) generalized dystonia. $\ddagger C=$ Of Caucasian descent (i.e. more than $90 \%$ of individuals in a data set belongs to this descent group); $\mathrm{A}=$ Asian descent; All = All ethnic groups combined (see Table S2 for study-specific details).

Figure 1. Flowchart of literature search, data extraction, and analysis strategies applied for the dystonia field synopsis. † Search for query terms "(dystoni* OR (writer* AND cramp) OR graphospasm OR blepharospasm OR torticollis OR meige OR spasmodic OR dysphonia OR retrocollis OR antecollis OR laterocollis OR (musician* AND cramp) OR (occupation* AND cramp) OR (golf* AND cramp) OR yips) AND associat* AND gene*”. $\ddagger$ Search for query terms outlined above and "AND ([gene name] OR [gene alias(es)])" using HGNC (HUGO Gene Nomenclature Committee at the European Bioinformatics Institute) nomenclature ${ }^{27}$. ๆ Only 45 out of 233 polymorphisms were investigated in at least two independent data sets. Furthermore, two studies were excluded due to sample overlap: 1. Djarmati et al, 2009 (ref. 19) fully excluded due to overlap with Lohman et al, 2012 (ref. 21), 2. Newman et al, 2012 (ref. 18) exclusion of data for polymorphisms overlapping with Newman et al, 2014 (ref. 20; see Table S2 for more details). 
bioRxiv preprint doi: https://doi.org/10.1101/306522; this version posted April 23,2018 . The copyright holder for this preprint (which was not certified by peer review) is the author/funder, who has granted bioRxiv a license to display the preprint in perpetuity. It is made available under aCC-BY-NC-ND 4.0 International license.

Ohlei et al, Genetic association studies in dystonia

\section{Supplementary Material (available from the authors upon request):}

Table S1. Overview of search algorithms on NCBI's "PubMed" database. In a first stage we searched for general keywords "(dystoni* OR (writer* AND cramp) OR graphospasm OR blepharospasm OR torticollis OR meige OR spasmodic OR dysphonia OR retrocollis OR antecollis OR laterocollis OR (musician* AND cramp) OR (occupation* AND cramp) OR (golf* AND cramp) OR yips) AND associat* AND gene*”. This resulted in 896 publications. In the second stage, we separately searched for each of the genes identified in stage $1(n=10)$ as well as all for 28 loci with a "DYT" label (supplement table 2; DYT designations taken from refs. 5 and 15). Each of these genes was searched for the different forms of isolated dystonia “(dystoni* OR (writer* AND cramp) OR graphospasm OR blepharospasm OR torticollis OR meige OR spasmodic OR dysphonia OR retrocollis OR antecollis OR laterocollis OR (musician* AND cramp) OR (occupation* AND cramp) OR (golf* AND cramp) OR yips)" in combination with their current official HGNC (HUGO Gene Nomenclature Committee at the European Bioinformatics Institute [28] (URL: https://www.genenames.org/) gene symbol as well as all previous symbols. "Some authors assign (COL6A3) as DYT2; here, this gene is listed under DYT27.

Table S2. Detailed summary of genetic association studies identified for this field synopsis and meta-analyses. Tabs correspond to diagnostic subgroups of dystonia; these were also utilized for the meta-analyses.

Table S3. Estimates of pairwise linkage disequilibrium between meta-analyzed SNPs within the same $1 \mathrm{Mb}$ interval, this only related to variants in PRKRA, DRD1, THAP1, TOR1A, DBH, DRD2, NACL, GCH1, ARSG and COMT based on genotype data from the 1000 genomes project, LD was calculated using PLINK v1.9. SNP pairs with $r^{2}>0.3$ were considered not independent. Bold font indicates $r^{2}>0.3$. 
Table 1. Results of random-effects meta-analyses based on an additive model in all types of isolated (a.k.a. "primary") dystonia.

\begin{tabular}{|c|c|c|c|c|c|c|c|c|}
\hline Chr-position & Gene & SNP & Allele & Ethnicity $\ddagger$ & $\begin{array}{l}\text { Cases vs Controls } \\
\text { (\# data sets/ \# publications) }\end{array}$ & OR $(95 \% \mathrm{Cl})$ & P-Value & $1^{2}$ \\
\hline $1: 236885200$ & MRT & rs1805087 & $G$ vs $A$ & C & 227 vs $1072(2 / 2)$ & $0.93(0.66-1.29)$ & 0.5852 & 0.00 \\
\hline 2:178433097 & PRKRA & rs6739142 & G vs $A$ & C & 357 vs $1200(2 / 2)$ & $0.99(0.73-1.36)$ & 0.9910 & 39.77 \\
\hline 2:178449632 & PRKRA & rs1967327 & C vs G & C & 357 vs $1200(2 / 2)$ & $1.10(0.86-1.42)$ & 0.4392 & 0.00 \\
\hline 2: 218326846 & PNKD & rs10932774 & $A$ vs $G$ & C & 357 vs $1200(2 / 2)$ & $1.02(0.84-1.24)$ & 0.8736 & 0.00 \\
\hline 3:114171968 & DRD3 & rs6280 & C vs T & C & 188 vs $200(2 / 2)$ & $0.82(0.61-1.11)$ & 0.1979 & 0.00 \\
\hline 5:175441837 & DRD1 & rs 155417 & T vs $\mathrm{C}$ & C & 188 vs $200(2 / 2)$ & $0.73(0.31-1.74)$ & 0.4792 & 0.00 \\
\hline 5:175443147 & DRD1 & rs4532 & C vs T & C & 315 vs $1172(3 / 3)$ & $1.37(1.13-1.67)$ & 0.0015 & 0.00 \\
\hline 7:94635805 & SGCE & rs10235385 & $C$ vs $A$ & C & 357 vs $1200(2 / 2)$ & $0.90(0.69-1.16)$ & 0.4028 & 0.00 \\
\hline 8:42838423 & THAP1 & rs111989331 & C vs T & All & 499 vs $360(2 / 2)$ & $0.69(0.31-1.54)$ & 0.3686 & 74.48 \\
\hline 8:42842898 & THAP1 & rs71521601 & G vs $A$ & C & 864 vs $510(3 / 3)$ & $1.20(0.66-2.21)$ & 0.5484 & 73.02 \\
\hline 8:42843330-1 & THAP1 & rs370983900 \& rs184497763 & $A A$ vs $C T$ & C & 3679 vs $2315(7 / 7)$ & $1.07(0.73-1.56)$ & 0.7431 & 40.61 \\
\hline $9: 129812583$ & TOR1A & rs13283584 & T vs C & C & 473 vs $749(2 / 2)$ & $1.32(1.08-1.61)$ & 0.0058 & 0.00 \\
\hline 9:129813148 & TOR1A & rs3842225 & - vs $C$ & All & 2287 vs $2503(11 / 10)$ & $1.05(0.91-1.21)$ & 0.4862 & 43.18 \\
\hline 9:129813148 & TOR1A & rs3842225 & - vs C & C & 1835 vs $2083(9 / 8)$ & $1.01(0.86-1.18)$ & 0.9450 & 44.67 \\
\hline 9:129813148 & TOR1A & rs3842225 & - vs C & A & 452 vs $420(2 / 2)$ & $1.28(0.99-0.65)$ & 0.0505 & 0.00 \\
\hline 9:129813558 & TOR1A & rs35153737 & - vs C & A & 452 vs $420(2 / 2)$ & $1.46(1.14-1.88)$ & 0.0031 & 0.00 \\
\hline 9:129813781 & TOR1A & rs1182 & $A$ vs $C$ & All & 2213 vs $2526(11 / 10)$ & $1.05(0.85-1.30)$ & 0.6742 & 72.36 \\
\hline 9:129813781 & TOR1A & rs1182 & $A$ vs $C$ & C & 1572 vs $2010(8 / 7)$ & $1.01(0.77-1.32)$ & 0.9607 & 79.00 \\
\hline 9:129813781 & TOR1A & rs1182 & $A$ vs $C$ & A & 641 vs $516(3 / 3)$ & $1.15(0.90-1.45)$ & 0.2647 & 0.00 \\
\hline 9:129816005 & TOR1A & rs 11787741 & $G$ vs $A$ & C & 473 vs $749(2 / 2)$ & $0.95(0.77-1.17)$ & 0.6260 & 0.00 \\
\hline 9:129818407 & TOR1A & rs13297609 & C vs G & All & 351 vs $428(2 / 2)$ & $1.16(0.89-1.52)$ & 0.2742 & 0.00 \\
\hline 9:129818622 & TOR1A & rs1801968 & G vs $C$ & All & 2075 vs $2699(11 / 11)$ & $0.95(0.78-1.16)$ & 0.6332 & 43.00 \\
\hline 9:129818622 & TOR1A & rs1801968 & G vs $C$ & C & 1428 vs $1925(6 / 6)$ & $1.02(0.87-1.20)$ & 0.7870 & 5.33 \\
\hline 9:129818622 & TOR1A & rs1801968 & G vs $C$ & A & 626 vs $494(4 / 4)$ & $0.74(0.44-1.26)$ & 0.2641 & 62.34 \\
\hline 9:129822779 & TOR1A & rs2296793 & $A$ vs $G$ & All & 1692 vs $2277(10 / 9)$ & $1.02(0.87-1.18)$ & 0.8381 & 42.61 \\
\hline 9:129822779 & TOR1A & rs2296793 & $A$ vs $G$ & C & 1454 vs $1946(8 / 7)$ & $1.02(0.86-1.21)$ & 0.8221 & 45.59 \\
\hline 9:129822779 & TOR1A & rs2296793 & $A$ vs $G$ & A & 238 vs $331(2 / 2)$ & $0.99(0.60-1.64)$ & 0.9738 & 64.39 \\
\hline 9:133636819 & DBH & rs2797849 & C vs $G$ & C & 490 vs 3054 (2/2) & $1.09(0.89-1.32)$ & 0.4079 & 38.64 \\
\hline 9:133639458 & $\mathrm{DBH}$ & rs2797851 & $A$ vs $G$ & C & 490 vs $3054(2 / 2)$ & $1.07(0.87-1.34)$ & 0.5395 & 42.38 \\
\hline 9:133657065 & $\mathrm{DBH}$ & rs1611131 & $G$ vs $A$ & C & 490 vs $3054(2 / 2)$ & $1.12(0.83-1.51)$ & 0.4528 & 69.85 \\
\hline 9:133657152 & $\mathrm{DBH}$ & rs6271 & T vs $\mathrm{C}$ & C & 490 vs $3054(2 / 2)$ & $2.97(0.37-24.02)$ & 0.3002 & 96.73 \\
\hline 9:133663599 & DBH & rs129886 & T vs C & C & 490 vs 3054 (2/2) & $1.01(0.86-1.20)$ & 0.8704 & 0.00 \\
\hline 10:28431147 & intergenic & rs1249277 & G vs $C$ & C & 464 vs 5515 (2/2) & $0.77(0.41-1.45)$ & 0.4215 & 89.34 \\
\hline 11:27658369 & BDNF & rs6265 & T vs C & All & 1591 vs $2780(7 / 7)$ & $1.09(0.98-1.21)$ & 0.1165 & 0.00 \\
\hline 11:27658369 & BDNF & rs6265 & T vs C & C & 1146 vs 2350 (5/5) & $1.05(0.91-1.21)$ & 0.4936 & 7.52 \\
\hline 11:27658369 & BDNF & rs6265 & T vs C & A & 445 vs 430 (2/2) & $1.19(0.98-1.43)$ & 0.0759 & 0.00 \\
\hline $11: 48246304$ & OR4X2 & rs67863238 & C vs G & C & 464 vs $5515(2 / 2)$ & $2.17(0.40-11.70)$ & 0.3482 & 96.50 \\
\hline
\end{tabular}


Ohlei et al, Genetic association studies in dystonia

\begin{tabular}{|c|c|c|c|c|c|c|c|c|}
\hline $11: 113400106$ & DRD2 & rs1800497 & $A$ vs $G$ & C & 222 vs $267(3 / 3)$ & $0.89(0.65-1.22)$ & 0.4758 & 0.00 \\
\hline $11: 113425564$ & DRD2 & rs1079597 & T vs C & C & 188 vs $200(2 / 2)$ & $1.00(0.69-1.46)$ & 0.9913 & 0.00 \\
\hline $11: 113475529$ & DRD2 & rs1799732 & - vs G & C & 188 vs $200(2 / 2)$ & $0.74(0.46-1.19)$ & 0.2064 & 0.00 \\
\hline 13:101406511 & NALCN & rs1338041 & $C$ vs $A$ & All & 565 vs $5804(3 / 3)$ & $0.90(0.59-1.36)$ & 0.6014 & 89.79 \\
\hline 13:101406511 & NALCN & rs1338041 & $C$ vs $A$ & C & 464 vs $5515(2 / 2)$ & $0.75(0.51-1.10)$ & 0.1445 & 83.78 \\
\hline 13:101407929 & NALCN & rs9518385 & $C$ vs $A$ & C & 339 vs $6145(2 / 2)$ & $0.73(0.51-1.05)$ & 0.0901 & 76.89 \\
\hline 13:101430922 & NALCN & rs61973742 & $G$ vs $A$ & All & 665 vs $5804(3 / 3)$ & $1.38(0.41-4.64)$ & 0.6001 & 96.35 \\
\hline 13:101430922 & NALCN & rs61973742 & $G$ vs $A$ & C & 464 vs $5515(2 / 2)$ & $1.85(0.26-13.37)$ & 0.5245 & 97.41 \\
\hline 14:54837362 & $\mathrm{GCH} 1$ & rs2145945 & $A$ vs $G$ & C & 357 vs $1200(2 / 2)$ & $0.92(0.66-1.27)$ & 0.6038 & 64.12 \\
\hline 14:54839739 & $\mathrm{GCH} 1$ & rs10483639 & $C$ vs $G$ & C & 360 vs $2062(2 / 2)$ & $1.09(0.72-1.64)$ & 0.6990 & 76.69 \\
\hline $14: 54841286$ & $\mathrm{GCH} 1$ & rs 17739146 & G vs $A$ & C & 357 vs $1200(2 / 2)$ & $0.91(0.66-1.27)$ & 0.5921 & 22.85 \\
\hline $14: 54888313$ & $\mathrm{GCH} 1$ & rs998259 & T vs C & C & 357 vs $1200(2 / 2)$ & $0.97(0.74-1.27)$ & 0.7973 & 36.39 \\
\hline $17: 68368608$ & ARSG & rs1558877 & T vs C & C & 357 vs $5272(2 / 2)$ & $0.96(0.78-1.18)$ & 0.6883 & 39.26 \\
\hline $17: 68368663$ & ARSG & rs1558878 & C vs T & C & 357 vs $5272(2 / 2)$ & $0.88(0.75-1.02)$ & 0.0846 & 0.00 \\
\hline $17: 68386068$ & ARSG & rs 11655081 & C vs T & All & 2420 vs $3608(9 / 1)$ & $1.33(0.95-1.86)$ & 0.0989 & 77.20 \\
\hline $17: 68386068$ & ARSG & rs11655081 & C vs T & C & 2243 vs $3409(8 / 1)$ & $1.38(0.92-2.08)$ & 0.1224 & 79.97 \\
\hline 17:68395091 & ARSG & rs7342975 & G vs $A$ & C & 357 vs $5272(2 / 2)$ & $2.00(1.15-3.47)$ & 0.0057 & 0.00 \\
\hline 17:68395135 & ARSG & rs9972951 & A vs $G$ & C & 357 vs $5272(2 / 2)$ & $2.16(1.06-4.40)$ & 0.0342 & 68.35 \\
\hline 22:19950115 & COMT & rs5993883 & T vs $G$ & C & 490 vs $3056(2 / 2)$ & $0.90(0.61-1.31)$ & 0.5671 & 83.57 \\
\hline 22:19964609 & COMT & rs4646316 & T vs C & C & 490 vs $3056(2 / 2)$ & $1.08(0.92-1.27)$ & 0.3332 & 0.00 \\
\hline 22:19968169 & COMT & rs9332377 & T vs C & C & 490 vs $3056(2 / 2)$ & $1.11(0.92-1.34)$ & 0.2678 & 0.00 \\
\hline
\end{tabular}

$\ddagger_{\mathrm{C}}=$ Of Caucasian descent (i.e. more than $90 \%$ of individuals in a data set belongs to this descent group); $\mathrm{A}=\mathrm{Asian}$ descent; All = All ethnic

groups combined (see Table S2 for study-specific details). 
Table 2. Results of random-effects meta-analyses based on an additive model in diagnostic subgroups of isolated dystonia: a) all types of focal dystonia, b) musician's dystonia, c) blepharospasm, d) cervical dystonia, e) spasmodic dystonia, f) writer's dystonia, g) segmental dystonia, h) generalized dystonia.

\section{A. All types of focal dystonia.}

\begin{tabular}{|c|c|c|c|c|c|c|c|c|}
\hline Chr-position & Gene & SNP & Allele & Ethnicity ${ }^{\ddagger}$ & $\begin{array}{l}\text { Cases vs Controls } \\
\text { (\# data sets/\# publications) }\end{array}$ & OR $(95 \% \mathrm{Cl})$ & P-Value & $\mathrm{I}^{2}$ \\
\hline $1: 236885200$ & MRT & rs1805087 & G vs $A$ & $\mathrm{C}$ & 227 vs $1072(2 / 2)$ & $0.93(0.66-1.29)$ & 0.5852 & 0.00 \\
\hline 3:114171968 & DRD3 & rs6280 & C vs T & C & 188 vs $200(2 / 2)$ & $0.82(0.61-1.11)$ & 0.1979 & 0.00 \\
\hline $5: 175441837$ & DRD1 & rs155417 & T vs $\mathrm{C}$ & C & 188 vs 200 (2/2) & $0.73(0.31-1.74)$ & 0.4792 & 0.00 \\
\hline $5: 175443147$ & DRD1 & rs4532 & C vs T & C & 315 vs $1172(3 / 3)$ & $1.37(1.13-1.67)$ & 0.0015 & 0.00 \\
\hline 8:42843330-1 & THAP1 & rs370983900 \& rs184497763 & AA vs CT & C & 1838 vs $1885(2 / 2)$ & $1.13(0.82-1.56)$ & 0.4483 & 0.00 \\
\hline 9:129813148 & TOR1A & rs3842225 & - vs C & C & 747 vs $906(4 / 3)$ & $1.09(0.92-1.29)$ & 0.3423 & 0.00 \\
\hline 9:129813781 & TOR1A & rs1182 & $A$ vs $C$ & All & 895 vs $1200(5 / 4)$ & $1.07(0.93-1.27)$ & 0.3105 & 0.00 \\
\hline $9: 129813781$ & TOR1A & rs1182 & $A$ vs $C$ & C & 747 vs $906(4 / 3)$ & $1.07(0.90-1.27)$ & 0.4348 & 0.00 \\
\hline $9: 129818622$ & TOR1A & rs1801968 & G vs $C$ & All & 1116 vs $1905(6 / 6)$ & $1.06(0.89-1.27)$ & 0.5114 & 5.33 \\
\hline 9:129818622 & TOR1A & rs1801968 & G vs $C$ & C & 889 vs $1674(4 / 4)$ & $1.07(0.87-1.31)$ & 0.5170 & 13.98 \\
\hline 9:129818622 & TOR1A & rs1801968 & G vs $C$ & A & 227 vs $231(2 / 2)$ & $0.99(0.55-1.80)$ & 0.9872 & 41.70 \\
\hline 9:129822779 & TOR1A & rs2296793 & $A$ vs $G$ & All & 729 vs $973(5 / 4)$ & $0.97(0.81-1.16)$ & 0.7446 & 14.35 \\
\hline 9:129822779 & TOR1A & rs2296793 & $A$ vs $G$ & C & 612 vs $842(4 / 3)$ & $1.01(0.83-1.23)$ & 0.9066 & 9.29 \\
\hline $9: 133636819$ & DBH & rs2797849 & $C$ vs $G$ & C & 490 vs $3054(2 / 2)$ & $1.09(0.89-1.32)$ & 0.4079 & 38.64 \\
\hline $9: 133639458$ & $\mathrm{DBH}$ & rs2797851 & $A$ vs $G$ & C & 490 vs $3054(2 / 2)$ & $1.07(0.87-1.34)$ & 0.5395 & 42.38 \\
\hline $9: 133657065$ & $\mathrm{DBH}$ & rs 1611131 & $G$ vs $A$ & C & 490 vs $3054(2 / 2)$ & $1.12(0.83-1.51)$ & 0.4528 & 69.85 \\
\hline 9:133657152 & DBH & rs6271 & T vs $C$ & C & 490 vs $3054(2 / 2)$ & $2.97(0.37-24.02)$ & 0.3002 & 96.73 \\
\hline $9: 133663599$ & DBH & rs129886 & T vs $\mathrm{C}$ & C & 490 vs $3054(2 / 2)$ & $1.01(0.86-1.20)$ & 0.8704 & 0.00 \\
\hline 10:28431147 & intergenic & rs1249277 & G vs $C$ & C & 464 vs $5515(2 / 2)$ & $0.77(0.41-1.45)$ & 0.4215 & 89.34 \\
\hline $11: 27658369$ & BDNF & rs6265 & T vs C & All & 739 vs $2030(4 / 4)$ & $1.13(0.91-1.41)$ & 0.2678 & 43.72 \\
\hline $11: 27658369$ & BDNF & rs6265 & T vs $C$ & C & 589 vs $1813(3 / 3)$ & $1.07(0.81-1.41)$ & 0.1689 & 43.77 \\
\hline $11: 48246304$ & OR4X2 & rs67863238 & C vs G & C & 464 vs 5515 (2/2) & $2.17(0.40-11.70)$ & 0.3482 & 96.50 \\
\hline $11: 113400106$ & DRD2 & rs 1800497 & $A$ vs $G$ & C & 222 vs $267(3 / 3)$ & $0.89(0.65-1.22)$ & 0.4758 & 0.00 \\
\hline $11: 113425564$ & DRD2 & rs1079597 & T vs C & C & 188 vs $200(2 / 2)$ & $1.00(0.69-1.46)$ & 0.9913 & 0.00 \\
\hline $11: 113475529$ & DRD2 & rs1799732 & - vs $G$ & $\mathrm{C}$ & 188 vs $200(2 / 2)$ & $0.74(0.46-1.19)$ & 0.2064 & 0.00 \\
\hline 13:101406511 & NALCN & rs1338041 & C vs $A$ & All & 665 vs $5804(3 / 3)$ & $0.90(0.59-1.36)$ & 0.6014 & 89.79 \\
\hline 13:101406511 & NALCN & rs1338041 & $C$ vs $A$ & C & 464 vs $5515(2 / 2)$ & $0.75(0.50-1.11)$ & 0.1445 & 83.78 \\
\hline 13:101407929 & NALCN & rs9518385 & $C$ vs $A$ & C & 339 vs $6145(2 / 2)$ & $0.73(0.51-1.05)$ & 0.0901 & 76.89 \\
\hline 13:101430922 & NALCN & rs61973742 & $G$ vs $A$ & All & 665 vs $5804(3 / 3)$ & $1.38(0.41-4.64)$ & 0.6001 & 96.35 \\
\hline 13:101430922 & NALCN & rs61973742 & $G$ vs $A$ & $\mathrm{C}$ & 464 vs $5515(2 / 2)$ & $1.85(0.26-13.37)$ & 0.5245 & 97.41 \\
\hline $17: 68368608$ & ARSG & rs1558877 & T vs $\mathrm{C}$ & C & 357 vs $5272(2 / 2)$ & $0.96(0.78-1.18)$ & 0.6883 & 39.26 \\
\hline $17: 68368663$ & ARSG & rs1558878 & C vs T & $\mathrm{C}$ & 357 vs $5272(2 / 2)$ & $0.88(0.75-1.02)$ & 0.0846 & 0.00 \\
\hline $17: 68386068$ & ARSG & rs11655081 & C vs T & All & 2007 vs $3608(9 / 1)$ & $1.34(0.93-1.94)$ & 0.1108 & 78.22 \\
\hline $17: 68386068$ & ARSG & rs11655081 & $\mathrm{C}$ vs T & C & 1877 vs $3409(8 / 1)$ & $1.41(0.91-2.20)$ & 0.1226 & 80.61 \\
\hline 17:68395091 & ARSG & rs7342975 & G vs $A$ & C & 357 vs $5272(2 / 2)$ & $2.00(1.15-3.47)$ & 0.0057 & 0.00 \\
\hline
\end{tabular}




\begin{tabular}{|c|c|c|c|c|c|c|c|c|}
\hline 17:68395135 & ARSG & rs9972951 & A vs $\mathrm{G}$ & C & 357 vs $5272(2 / 2)$ & $2.16(1.06-4.40)$ & 0.0342 & 68.35 \\
\hline 22:19950115 & COMT & rs5993883 & T vs $\mathrm{G}$ & C & 490 vs $3056(2 / 2)$ & $0.90(0.61-1.31)$ & 0.5671 & 83.57 \\
\hline 22:19964609 & COMT & rs4646316 & T vs C & C & 490 vs $3056(2 / 2)$ & $1.08(0.92-1.27)$ & 0.3332 & 0.00 \\
\hline 22:19968169 & COMT & rs9332377 & T vs C & C & 490 vs $3056(2 / 2)$ & $1.11(0.92-1.34)$ & 0.2678 & 0.00 \\
\hline
\end{tabular}

\section{B. Musician's dystonia.}

\begin{tabular}{|c|c|c|c|c|c|c|c|c|}
\hline Chr-position & Gene & SNP & Allele & Ethnicity ${ }^{\ddagger}$ & $\begin{array}{l}\text { Cases vs Controls } \\
\text { (\# data sets/\# publications) }\end{array}$ & OR $(95 \% \mathrm{Cl})$ & P-Value & $\mathrm{I}^{2}$ \\
\hline 9:129818622 & TOR1A & rs1801968 & G vs $C$ & $\mathrm{C}$ & 239 vs $1213(2 / 2)$ & $0.79(0.58-1.08)$ & 0.1522 & 0.00 \\
\hline $17: 68368608$ & ARSG & rs1558877 & T vs C & $\mathrm{C}$ & 258 vs $5272(2 / 2)$ & $0.93(0.69-1.25)$ & 0.6176 & 67.05 \\
\hline $17: 68368663$ & ARSG & rs1558878 & C vs T & $\mathrm{C}$ & 258 vs $5272(2 / 2)$ & 0.87() $0.73-1.05)$ & 0.1464 & 12.22 \\
\hline $17: 68386068$ & ARSG & rs11655081 & C vs T & C & 243 vs $1097(2 / 1)$ & $4.42(2.72-7.19)$ & 2.11e-09 & 0.00 \\
\hline 17:68395091 & ARSG & rs7342975 & $G$ vs $A$ & $\mathrm{C}$ & 258 vs $5272(2 / 2)$ & $2.00(1.15-3.47)$ & 0.0141 & 0.00 \\
\hline $17: 68395135$ & ARSG & rs9972951 & A vs $\mathrm{G}$ & C & 258 vs $5272(\mathbf{2} / \mathbf{2})$ & $2.16(1.02-4.55)$ & 0.0425 & 66.99 \\
\hline
\end{tabular}

\section{Blepharospasm.}

\begin{tabular}{|c|c|c|c|c|c|c|c|c|}
\hline Chr-position & Gene & SNP & Allele & Ethnicity ${ }^{\ddagger}$ & $\begin{array}{l}\text { Cases vs Controls } \\
\text { (\# data sets/\# publications) }\end{array}$ & OR $(95 \% \mathrm{Cl})$ & P-Value & $\mathrm{I}^{2}$ \\
\hline 8:42843331 & THAP1 & rs370983900 \& rs184497763 & AA vs CT & C & 228 vs $1885(2 / 2)$ & $0.80(0.39-1.65)$ & 0.5470 & 3.80 \\
\hline $9: 129813148$ & TOR1A & rs3842225 & - vs C & C & 205 vs $381(2 / 1)$ & $1.10(0.70-1.72)$ & 0.6807 & 52.83 \\
\hline 9:129813781 & TOR1A & rs1182 & A vs $C$ & All & 227 vs $675(3 / 2)$ & $1.12(0.84-1.49)$ & 0.4261 & 0.00 \\
\hline 9:129813781 & TOR1A & rs1182 & $A$ vs $C$ & C & 205 vs $381(2 / 1)$ & $1.08(0.73-1.59)$ & 0.7185 & 38.15 \\
\hline 9:129818622 & TOR1A & rs1801968 & G vs $C$ & All & 125 vs $472(3 / 2)$ & $1.21(0.60-2.44)$ & 0.5994 & 53.92 \\
\hline 9:129818622 & TOR1A & rs1801968 & G vs $C$ & A & 94 vs $231(2 / 2)$ & $0.91(0.38-2.19)$ & 0.8377 & 44.02 \\
\hline 9:129822779 & TOR1A & rs2296793 & $A$ vs $G$ & All & 281 vs 512 (3/2) & $0.85(0.66-1.09)$ & 0.2000 & 0.00 \\
\hline $9: 129822779$ & TOR1A & rs2296793 & $A$ vs $G$ & C & 205 vs $381(2 / 1)$ & $0.87(0.65-1.16)$ & 0.3309 & 0.00 \\
\hline $11: 27658369$ & BDNF & rs6265 & T vs $\mathrm{C}$ & All & 289 vs 1004 (2/2) & $1.44(0.76-2.71)$ & 0.2634 & 80.88 \\
\hline $17: 68386068$ & ARSG & rs11655081 & C vs T & C & 303 vs $1020(3 / 1)$ & $0.91(0.63-1.33)$ & 0.6372 & 0.00 \\
\hline
\end{tabular}




\section{Cervical dystonia.}

\begin{tabular}{|c|c|c|c|c|c|c|c|c|}
\hline Chr-position & Gene & SNP & Allele & Ethnicity ${ }^{\ddagger}$ & $\begin{array}{l}\text { Cases vs Controls } \\
\text { (\# data sets/\# publications) }\end{array}$ & OR $(95 \% \mathrm{Cl})$ & P-Value & $1^{2}$ \\
\hline $8: 42843331$ & THAP1 & rs370983900 \& rs184497763 & AA vs CT & $\mathrm{C}$ & 712 vs $1885(2 / 2)$ & $1.12(0.69-1.82)$ & 0.6472 & 22.08 \\
\hline 9:129813781 & TOR1A & rs1182 & A vs $C$ & All & 448 vs $655(2 / 2)$ & $1.03(0.82-1.29)$ & 0.787 & 0.00 \\
\hline $9: 129818622$ & TOR1A & rs1801968 & G vs $C$ & All & 570 vs $833(4 / 4)$ & $1.19(0.95-1.49)$ & 0.1276 & 0.00 \\
\hline $9: 129818622$ & TOR1A & rs1801968 & G vs $C$ & C & 478 vs $602(2 / 2)$ & $1.27(0.92-1.75)$ & 0.1502 & 38.78 \\
\hline $9: 129818622$ & TOR1A & rs1801968 & $G$ vs $C$ & A & 92 vs $231(2 / 2)$ & $0.93(0.51-1.71)$ & 0.8158 & 0.00 \\
\hline 9:129822779 & TOR1A & rs2296793 & $A$ vs $G$ & All & 399 vs $492(2 / 2)$ & $1.00(0.79-1.26)$ & 0.9953 & 0.00 \\
\hline 10:28431147 & intergenic & rs1249277 & G vs $C$ & $\mathrm{C}$ & 464 vs $5515(2 / 2)$ & $0.77(0.41-1.45)$ & 0.4215 & 89.34 \\
\hline 11:27658369 & BDNF & rs6265 & T vs C & All & 289 vs $1004(2 / 2)$ & $1.04(0.84-1.29)$ & 0.7138 & 0.00 \\
\hline $11: 48246304$ & OR4X2 & rs67863238 & C vs G & $\mathrm{C}$ & 464 vs $5515(2 / 2)$ & $2.17(0.40-11.70)$ & 0.3482 & 96.50 \\
\hline 13:101406511 & NALCN & rs1338041 & C vs $A$ & All & 665 vs $5804(3 / 3)$ & $0.90(0.59-1.36)$ & 0.6014 & 89.79 \\
\hline 13:101406511 & NALCN & rs1338041 & C vs A & $\mathrm{C}$ & 464 vs 5515 (2/2) & $0.75(0.51-1.10)$ & 0.1445 & 83.78 \\
\hline 13:101430922 & NALCN & rs61973742 & $G$ vs $A$ & All & 665 vs $5804(3 / 3)$ & $1.38(0.41-4.64)$ & 0.6001 & 96.35 \\
\hline 13:101430922 & NALCN & rs61973742 & $G$ vs $A$ & C & 464 vs $5515(2 / 2)$ & $1.85(0.26-13.37)$ & 0.5245 & 97.41 \\
\hline $17: 68386068$ & ARSG & rs 11655081 & C vs T & All & 1051 vs $2233(6 / 1)$ & $1.01(0.82-1.23)$ & 0.9456 & 0.00 \\
\hline $17: 68386068$ & ARSG & rs11655081 & C vs T & $\mathrm{C}$ & 961 vs $2034(5 / 1)$ & $0.97(0.76-1.24)$ & 0.8145 & 0.00 \\
\hline
\end{tabular}

\section{E. Spasmodic dystonia.}

\begin{tabular}{|c|c|c|c|c|c|c|c|c|}
\hline Chr-position & Gene & SNP & Allele & Ethnicity ${ }^{\ddagger}$ & $\begin{array}{l}\text { Cases vs Controls } \\
\text { (\# data sets/\# publications) }\end{array}$ & OR $(95 \% \mathrm{Cl})$ & P-Value & $\mathrm{I}^{2}$ \\
\hline $8: 4284333$ & THAP1 & rs370983900 \& rs184497763 & AA vs TC & $\mathrm{C}$ & 482 vs $1885(2 / 2)$ & $1.47(0.92-2.35)$ & 0.1040 & 0.00 \\
\hline
\end{tabular}

\section{F. Writer's cramp.}

\begin{tabular}{|c|c|c|c|c|c|c|c|c|}
\hline Chr-position & Gene & SNP & Allele & Ethnicity $^{\ddagger}$ & $\begin{array}{l}\text { Cases vs Controls } \\
\text { (\# data sets/\# publications) }\end{array}$ & OR (95\%Cl) & P-Value & $1^{2}$ \\
\hline $9: 129818622$ & TOR1A & rs1801968 & G vs C & C & 55 vs $341(2 / 1)$ & $3.10(1.25-7.68)$ & 0.01421 & 65.44 \\
\hline
\end{tabular}




\section{G. Segmental dystonia.}

\begin{tabular}{|c|c|c|c|c|c|c|c|c|}
\hline Chr-position & Gene & SNP & Allele & Ethnicity ${ }^{\ddagger}$ & $\begin{array}{l}\text { Cases vs Controls } \\
\text { (\# data sets/ \# publications) }\end{array}$ & OR $(95 \% \mathrm{Cl})$ & P-Value & $1^{2}$ \\
\hline $8: 42843331$ & THAP1 & rs370983900 \& rs184497763 & AA vs TC & $\mathrm{C}$ & 267 vs $1885(2 / 2)$ & $1.09(0.59-2.03)$ & 0.7765 & 0.00 \\
\hline $17: 68386068$ & ARSG & rs11655081 & C vs T & All & 344 vs $1219(4 / 1)$ & $1.06(0.80-1.40)$ & 0.6835 & 0.00 \\
\hline $17: 68386068$ & ARSG & rs11655081 & C vs T & $\mathrm{C}$ & 297 vs $1020(3 / 1)$ & $1.10(0.76-1.59)$ & 0.6078 & 6.72 \\
\hline
\end{tabular}

\section{H. Generalized dystonia in all populations.}

\begin{tabular}{|c|c|c|c|c|c|c|c|c|}
\hline Chr-position & Gene & SNP & Allele & Ethnicity $^{\ddagger}$ & $\begin{array}{l}\text { Cases vs Controls } \\
\text { (\# data sets/\# publications) }\end{array}$ & OR (95\%Cl) & P-Value & $1^{2}$ \\
\hline $9: 129818622$ & THAP1 & rs370983900 \& rs184497763 & AA vs CT & C & 99 vs $1885(2 / 2)$ & $0.85(0.30-2.43)$ & 0.7672 & 0.00 \\
\hline
\end{tabular}

${ }_{\ddagger} \mathrm{C}=$ Of Caucasian descent (i.e. more than $90 \%$ of individuals in a data set belongs to this descent group); $A=A$ sian descent; $A l l=A l l$ ethnic groups combined (see Table S2 for study-specific details). 
Figure 1. Flowchart of literature search, data extraction, and analysis strategies applied for the dystonia field synopsis.

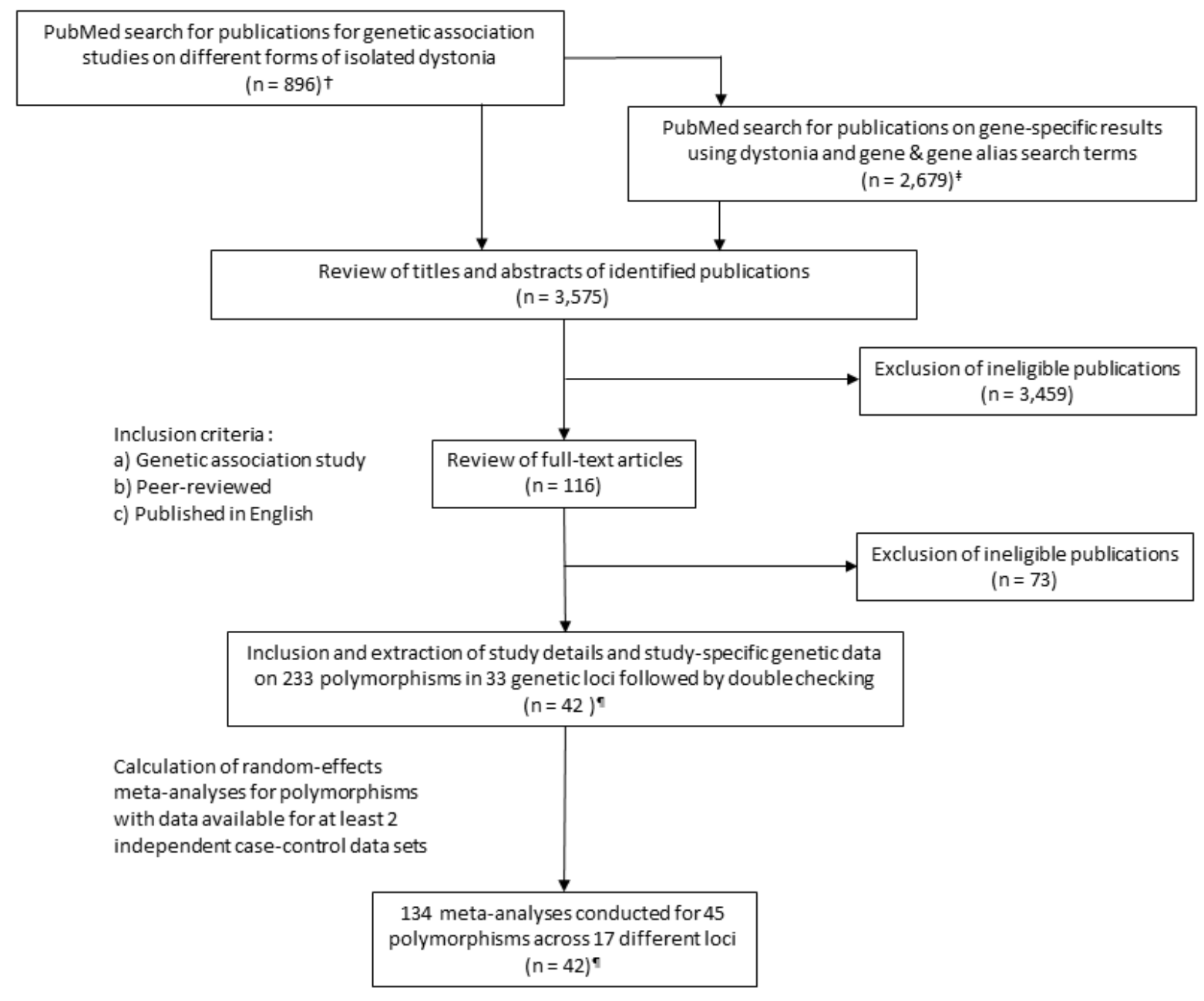

